Revista Brasil. Bot., V.31, n.3, p.377-388, jul.-set. 2008

\title{
Influence of soil properties on the abundance of plant species in ferruginous rocky soils vegetation, southeastern Brazil ${ }^{1}$
}

\author{
REGINA DE CASTRO VINCENT ${ }^{2,4}$ and MARICO MEGURO ${ }^{3}$
}

(received: November 03, 2005; accepted: May 29, 2008)

\begin{abstract}
Influence of soil properties on the abundance of plant species in ferruginous rocky soils vegetation, southeastern Brazil). Ferruginous "campos rupestres" are a particular type of vegetation growing on iron-rich primary soils. We investigated the influence of soil properties on plant species abundance at two sites of ferruginous "campos rupestres" and one site of quartzitic "campo rupestre", all of them in "Quadrilátero Ferrífero", in Minas Gerais State, southeastern Brazil. In each site, 30 quadrats were sampled to assess plant species composition and abundance, and soil samples were taken to perform chemical and physical analyses. The analyzed soils are strongly acidic and presented low fertility and high levels of metallic cations; a principal component analysis of soil data showed a clear segregation among sites due mainly to fertility and heavy metals content, especially $\mathrm{Cu}, \mathrm{Zn}$, and $\mathrm{Pb}$. The canonical correspondence analysis indicated a strong correlation between plant species abundance and soil properties, also segregating the sites.
\end{abstract}

Key words - ferruginous "campo rupestre", metalliferous soil, plant-soil relationships, "Quadrilátero Ferrífero"

RESUMO - (Influência das propriedades do solo sobre a abundância das espécies vegetais em campos ferruginosos no sudeste do Brasil). Os campos ferruginosos são um tipo particular de vegetação que cresce sobre solos primários ricos em ferro. Estudou-se a influência do solo sobre a abundância em espécies vegetais em duas áreas de campos ferruginosos e uma área de campo rupestre quartzítico, todos no Quadrilátero Ferrífero, em Minas Gerais, sudeste do Brasil. Em cada área foram amostradas 30 parcelas para avaliar a composição florística e a abundância, e analisar o solo. Os solos das áreas estudadas são fortemente ácidos, com baixa fertilidade e altos níveis de cátions metálicos, e mostraram clara separação na análise de componentes principais, devido principalmente à fertilidade e ao teor de metais pesados, especialmente $\mathrm{Cu}, \mathrm{Zn}$ e $\mathrm{Pb}$. A análise de correspondência canônica indicou uma forte correlação entre a abundância e as propriedades do solo, também separando as áreas.

Palavras-chave - campos rupestres ferruginosos, Quadrilátero Ferrífero, relações planta-solo, solo metalífero

\section{Introduction}

Ferruginous "campos rupestres" grow on "canga", a rock consisting of fragments of iron-formation and hard hematite cemented by limonite, an hydrated iron oxide (Simmons 1960, 1968, Pomerene 1964, Rizzini 1997). In Brazil, they are found mainly in a region known as "Quadrilátero Ferrífero", in Minas Gerais State (southeastern Brazil), and in "Serra dos Carajás", in Pará State (northern Brazil), sites that hold the main Brazilian iron ores. In literature, this vegetation was also called "canga vegetation" (Secco \& Mesquita 1983, Morellato \& Rosa 1991, Silva 1991, 1992), "campos rupestres" or rocky fields (Silva \& Rosa 1990, Silva et al. 1996) or ferruginous fields (Rizzini 1997, Vincent et al. 2002).

\footnotetext{
1. This work is part of a PhD thesis developed at the University of São Paulo, Departamento de Ecologia, São Paulo, SP, Brazil.

2. Secretaria Estadual do Meio Ambiente, DAIA, Av. Prof. Frederico Hermann Jr., 345, 05489-900 São Paulo, SP, Brazil.

3. Universidade de São Paulo, Instituto de Biociências, Departamento de Ecologia, Caixa Postal 11461, 05422-970 São Paulo, SP, Brazil.

4._Corresponding author: regina_c_vincent@yahoo.com.br
}

Since the term "campo rupestre" historically defines fields growing on quartzitic substrates (Magalhães 1966, Giulietti et al. 1987, 1997), we recommend the specification of the geological substrate for other "campos rupestres". In Minas Gerais, ferruginous "campos rupestres" occur mainly in the "cerrado" Province, a phytogeographical region characterized by a savannic vegetation with a physiognomic-floristic gradient varying from "campo sujo", a grassland with scattered shrubs, to a forest with more or less closed canopy, the "cerradão" (Coutinho 1978). Ferruginous "campos rupestres" of Minas Gerais hold many elements of "cerrado" and quartzitic "campo rupestre" flora and the families with more species are characteristic of the Espinhaço Range (Jacobi et al. 2007, Viana \& Lombardi 2007).

Rocky substrates and their derived primary soils may impose particular constraints to plant establishment, including soil scarcity, low water content, and low levels of nutrient. Additionally, high levels of heavy metals in metalliferous soils promote selection of resistant plant species that may show physiological and/or morphological adaptations (Porto \& Silva 1989, Silva \& Rosa 1990). 
In this type of substrate there are ecotypes adapted to high metal concentration in soil (Duvigneaud \& DenaeyerDe Smet 1960, Antonovics et al. 1971, Porto 1989, Köhl 1997), a low number of plant species and the presence of endemic species (Porto \& Silva 1989, Silva \& Rosa 1990). The metallophile vegetation is characterized by individuals with high concentrations of heavy metals in their tissues and occasionally by dwarf or giant ecotypes (Porto \& Silva 1989). The presence of high content of metal cations on soils is expected to influence phytosociological parameters of some populations (Howard-Williams 1970, Antonovics et al. 1971).

There are few studies concerning vegetation-soil heavy metal relationships in Brazilian metalliferous "campos rupestres". Heavy metal accumulation in plant tissues was studied in "Serra dos Carajás", Pará State (Porto \& Silva 1989, Silva 1992), "Quadrilátero Ferrífero", Minas Gerais State (Porto \& Silva 1989, Teixeira \& Lemos-Filho 1998), on ferruginous soils, and in ultramafic soils in Niquelândia, Goiás State (central Brazil, Reeves et al. 2007). Mining activities promote the destruction of this still poorly known ecosystem, suppressing plant species or populations that evolved in such a particular environment. The aims of present study were to investigate the influence of chemical and physical soil properties on plant species abundance in ferruginous "campos rupestres" and to compare them to a quartzitic "campo rupestre" in "Quadrilátero Ferrífero".

\section{Material and methods}

Study sites - The "Quadrilátero Ferrífero" is located in the southern portion of the "Cadeia do Espinhaço", in Minas

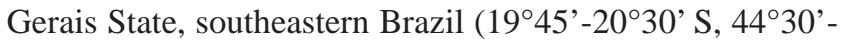
$43^{\circ} 07^{\prime} \mathrm{W}$; figure 1). The climate is Cwb of Köppen, with a dry season from April to September (Herz 1978). In the region, there are four types of physiognomies, depending on the geological nature of substrate: "campos cerrados" and quartzitic, granitic, and ferruginous "campos rupestres" (Giulietti et al. 1997, Rizzini 1997, Drummond et al. 2005). Ferruginous "campos rupestres" may develop on iron-rich rocky outcrops (locally known as "canga couraçada") and iron-rich stony soils ("canga nodular"), and the variation of their physiognomies depends on the degree of weathering of the same type of rock (Rizzini 1997, Vincent et al. 2002).

This study was carried out in the "Parque Estadual da Serra do Rola-Moça" (PESRM) and in its vicinity, in northwestern portion of "Quadrilátero Ferrífero" (figure 1), in the municipalities of Belo Horizonte, Brumadinho, Ibirité, and Nova Lima, in Minas Gerais State, Brazil. This park and vicinities include "cerrado" physiognomies (Brazilian savanna) in altitudes below $1,000 \mathrm{~m}$, riparian forests along valleys, and ferruginous and quartzitic "campos rupestres" above $1,000 \mathrm{~m}$. In order to understand the relationship between vegetation and soil properties in ferruginous "campos rupestres", we studied two areas of canga substrate and one of quartzitic "campo rupestre" as a control study site: a. "Canga couraçada" (CCo): this is a "campo sujo" growing on ferruginous rocky outcrop and situated on Serra da Mutuca $\left(20^{\circ} 01^{\prime} \mathrm{S}\right.$ and $\left.43^{\circ} 59^{\prime} \mathrm{W} ; 1,350 \mathrm{~m}\right)$ in PESRM. Shrubs grow on crevices and herbs grow on soil islands or directly on the rocks (epilithic plants). b. "Canga nodular" (CNo): this is a "campo sujo" growing on ferruginous stony (nodular) soil and situated on "Serra do Rola-Moça" ( $20^{\circ} 03^{\prime} \mathrm{S}$ and $44^{\circ} 01^{\prime} \mathrm{W}$; $1,350 \mathrm{~m}$ ) in PESRM. This field is composed mainly by grasses and sedges mixed with shrubs and subshrubs and with a sparse population of a shrubby Velloziaceae (Vellozia compacta Mart. ex Schult. f.). c. Quartzitic "campo rupestre" (QCR): this is a grassland growing on nodular quartzitic soils intermixed by sparse quartzitic outcrops and situated on "Serra da Calçada" $\left(20^{\circ} 06^{\prime} \mathrm{S}\right.$ and 4359' W; 1,300 m), Retiro das Pedras, near PESRM. It shows a "campo limpo" physiognomy, i.e., a grassland composed mainly by grasses and sedges. Ferruginous "campos rupestres" surround this area.

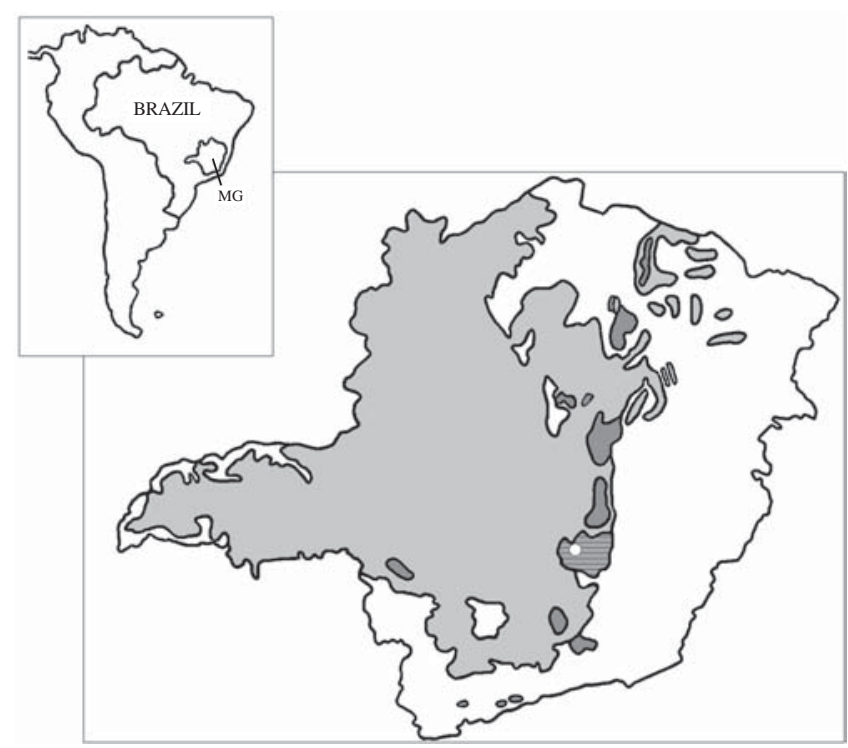

Figure 1. Distribution of "campos rupestres" above 1,000 m ( $\square$ ) and "cerrado" vegetation ( $\square$ ) in Minas Gerais State (MG), southeastern Brazil. The three study sites ( $\mathrm{O})$ are located inside "Quadrilátero Ferrífero" (目). Modified from PRODEMGE (1996).

Sampling - Fieldwork was conducted between December 2000 and June 2002. At each study site one area of $50 \times 50 \mathrm{~m}$ was delimited, where 30 quadrats $(2 \times 1 \mathrm{~m})$ were randomly established. In QCR we used quadrats of $1.0 \times 0.5 \mathrm{~m}$ due to the very high number of individuals found in a preliminary sampling when no strong difference on the number of 
recorded species was verified. All plants in the quadrats were recorded and determined to the species level when possible; individuals were defined as a unit emerging from soil. The voucher specimens were deposited in the herbaria of the "Universidade Federal de Minas Gerais" (BHCB) and "Escola Superior de Agricultura Luiz de Queiroz" (ESA). We used absolute density as measure of abundance (MuellerDombois \& Ellenberg 1974) due to the difference in quadrat size among sites.

Three soil samples (0-10 cm deep) were collected within each quadrat and then mixed, totalling 30 samples by site. The soil samples were air-dried, sieved in $2 \mathrm{~mm}$ sieve, and the fine and coarse fractions were weighted to calculate the proportion of "fine soil" (FS) in the substrate. Chemical and physical analyses $-\mathrm{pH}$, organic matter $(\mathrm{OM}), \mathrm{P}$, exchangeable $\mathrm{K}^{+}, \mathrm{Ca}^{2+}$ and $\mathrm{Mg}^{2+}, \mathrm{H}^{+}+\mathrm{Al}^{3+}(\mathrm{H}+\mathrm{Al})$, sum of bases (SB), cation exchange capacity (CEC), base saturation (V), B, sand, silt and clay proportions, and the levels of metallic cations $\mathrm{Cu}$, $\mathrm{Fe}, \mathrm{Mn}$, and $\mathrm{Zn}$ - were made at soil laboratories of "Escola Superior de Agricultura Luiz de Queiroz" (ESALQ-USP), while the determinations of metallic cations $\mathrm{Cd}, \mathrm{Cr}, \mathrm{Ni}$, and $\mathrm{Pb}$ were made at "Instituto Agronômico de Campinas" (IAC).

Data analysis - Soil variables were tested for normality by Shapiro-Wilk test $(P<0.05)$ and for homogeneity of variances by Levene test $(P<0.05)$. Since soil variables didn't have fulfilled parametric assumptions, multiple comparison tests were performed among the three study sites with KruskalWallis one-way ANOVA to detect significant differences $(P$ $<0.05$ ) (Siegel 1956, Zar 1996).

A principal component analysis (PCA) was performed with soil variables in the 90 quadrats to verify the segregation among study sites and to identify the variables most strongly correlated to groups (ter Braak 1995). Sum of bases and base saturation were not included due to their strong correlation with the other variables.

A canonical correspondence analysis (CCA) was performed to investigate the relationships between species abundance and soil variables in the 90 quadrats. Absolute density values were log-transformed after to add the value 1 to the original values to avoid problems with zero values (ter Braak 1995, ter Braak \& Looman 1995, Oliveira-Filho et al. 2001). Only species with $n \geq 10$ individuals and with occurrence in more than three of the 90 quadrats were included in the analysis. After a preliminary analysis, six variables were excluded due to high collinearity with other variables $-\mathrm{pH}$ and base saturation - or low correlation with the ordination axes $(-0.4<r<0.4)$ - sum of bases, $\mathrm{Cr}$ and $\mathrm{Ni}$ levels, and fine soil proportion. The programs used were PCOrd (McCune \& Mefford 1999) for ordination analyses and Statistica 6 (StatSoft, Inc. 2001) for tests of homogeneity of variances, normality and multiple comparisons.

\section{Results}

Soil properties - The soils of study sites are strongly acidic, and present low levels of $\mathrm{P}$, low base saturation, high levels of $\mathrm{Ca}, \mathrm{Fe}, \mathrm{Zn}$ and $\mathrm{Pb}$, and the substrate has a high proportion of coarse soil ( $\geq 2 \mathrm{~mm})$ (table 1$)$.

The sites were clearly discriminated in the PCA diagram (figure 2), where the two first axes explained $65.3 \%$ of total variance (Axis $1=39.0 \%$, Axis $2=26.3 \%$ ). The first axis was strongly positively correlated to CEC, $\mathrm{OM}, \mathrm{Fe}$ and $\mathrm{P}$ levels, and negatively correlated to $\mathrm{Mg}$, $\mathrm{pH}, \mathrm{Mn}$, and $\mathrm{Pb}$; this axis separates $\mathrm{CCo}$ from $\mathrm{CNo}$ and QCR, reflecting differences between rocky outcrop and

Table 1. Physical and chemical soil properties of "canga couraçada" (CCo) and "canga nodular" (CNo) in "Parque Estadual da Serra do Rola-Moça" and of quartzitic "campo rupestre" (QCR) in "Retiro das Pedras", southeastern Brazil. OM = organic matter; $\mathrm{H}+\mathrm{Al}=$ potential acidity; $\mathrm{SB}=$ sum of bases; $\mathrm{CEC}=$ cation exchange capacity; $\mathrm{V}$ = base saturation; $\mathrm{FS}=$ fine soil. Different letters after mean $(\bar{x})$ and standard deviation $(s)$ indicate significant differences in Kruskal-Wallis test $(P<0.05)$.

\begin{tabular}{|c|c|c|c|c|}
\hline & $\operatorname{CCo}(n=30)$ & $\mathrm{CNo}(n=30)$ & \multicolumn{2}{|c|}{ QCR $(n=30)$} \\
\hline & $\bar{x}$ & $\bar{x}$ & $\bar{x}$ & $s$ \\
\hline $\mathrm{pH}\left(\right.$ in $\left.\mathrm{CaCl}_{2}\right)$ & $3.43 \pm 0.12 \mathrm{c}$ & $4.38 \pm 0.22 \mathrm{a}$ & $4.01 \pm$ & $\pm \quad 0.10 \mathrm{~b}$ \\
\hline $\mathrm{OM}\left(\mathrm{g} \mathrm{dm}^{-3}\right)$ & $136.07 \pm 18.97 \mathrm{a}$ & $83.17 \pm 9.98 \mathrm{~b}$ & $39.83 \pm$ & $\pm \quad 4.58 \mathrm{c}$ \\
\hline $\mathrm{P}\left(\mathrm{mg} \mathrm{dm}^{-3}\right)$ & $14.83 \pm 2.51 \mathrm{a}$ & $10.23 \pm 1.17 \mathrm{~b}$ & $5.20 \pm$ & $\pm 1.03 \mathrm{c}$ \\
\hline $\mathrm{K}\left(\mathrm{mmol}_{\mathrm{c}} \mathrm{dm}^{-3}\right)$ & $1.93 \pm 0.30 \mathrm{~b}$ & $1.80 \pm 0.30 \mathrm{~b}$ & $3.98 \pm$ & $\pm \quad 1.29 \mathrm{a}$ \\
\hline $\mathrm{Ca}\left(\mathrm{mmol}_{\mathrm{c}} \mathrm{dm}^{-3}\right)$ & $14.80 \pm 5.03 \mathrm{~b}$ & $26.07 \pm 6.31 \mathrm{a}$ & $18.23 \pm$ & $\pm 4.61 \mathrm{~b}$ \\
\hline $\operatorname{Mg}\left(\mathrm{mmol}_{\mathrm{c}} \mathrm{dm}^{-3}\right)$ & $2.80 \pm 0.81 \mathrm{~b}$ & $5.30 \pm 1.24 \mathrm{a}$ & $5.48 \pm$ & $\pm \quad 1.19 \mathrm{a}$ \\
\hline $\mathrm{H}+\mathrm{Al}\left(\mathrm{mmol}_{\mathrm{c}} \mathrm{dm}^{-3}\right)$ & $247.07 \pm 30.29 a$ & $86.83 \pm 17.46 \mathrm{~b}$ & $72.53 \pm$ & $\pm 9.58 \mathrm{c}$ \\
\hline $\mathrm{SB}\left(\mathrm{mmol}_{\mathrm{c}} \mathrm{dm}^{-3}\right)$ & $19.53 \pm 5.82 \mathrm{c}$ & $33.17 \pm 7.56 \mathrm{a}$ & $27.43 \pm$ & $\pm 6.55 \mathrm{~b}$ \\
\hline $\mathrm{CEC}\left(\mathrm{mmol}_{\mathrm{c}} \mathrm{dm}^{-3}\right)$ & $266.60 \pm 31.35 \mathrm{a}$ & $120.00 \pm 14.64 \mathrm{~b}$ & $102.28 \pm$ & $\pm 18.40 \mathrm{c}$ \\
\hline $\mathrm{V}(\%)$ & $7.33 \pm 2.09 \mathrm{~b}$ & $28.03 \pm 6.98 \mathrm{a}$ & $28.37 \pm$ & $\pm 7.28 \mathrm{a}$ \\
\hline
\end{tabular}


continuation

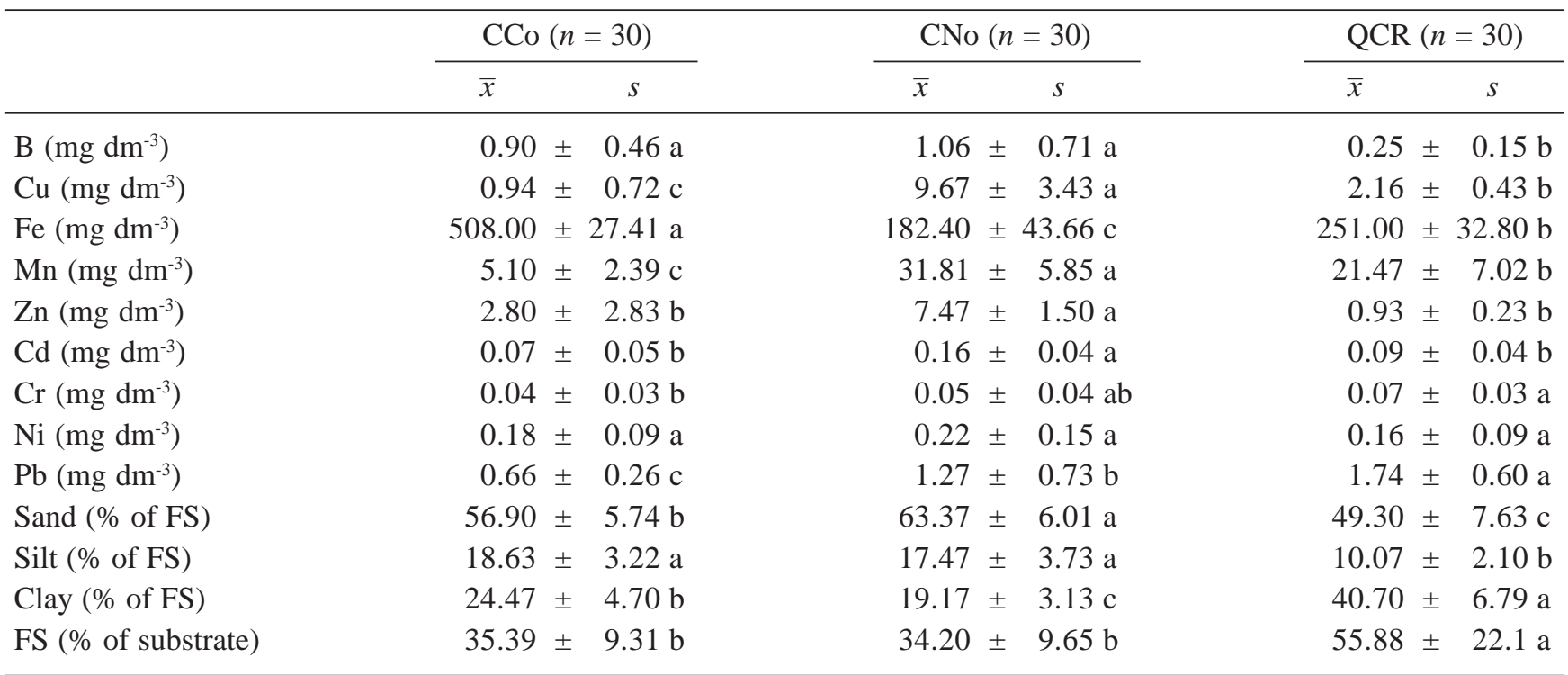

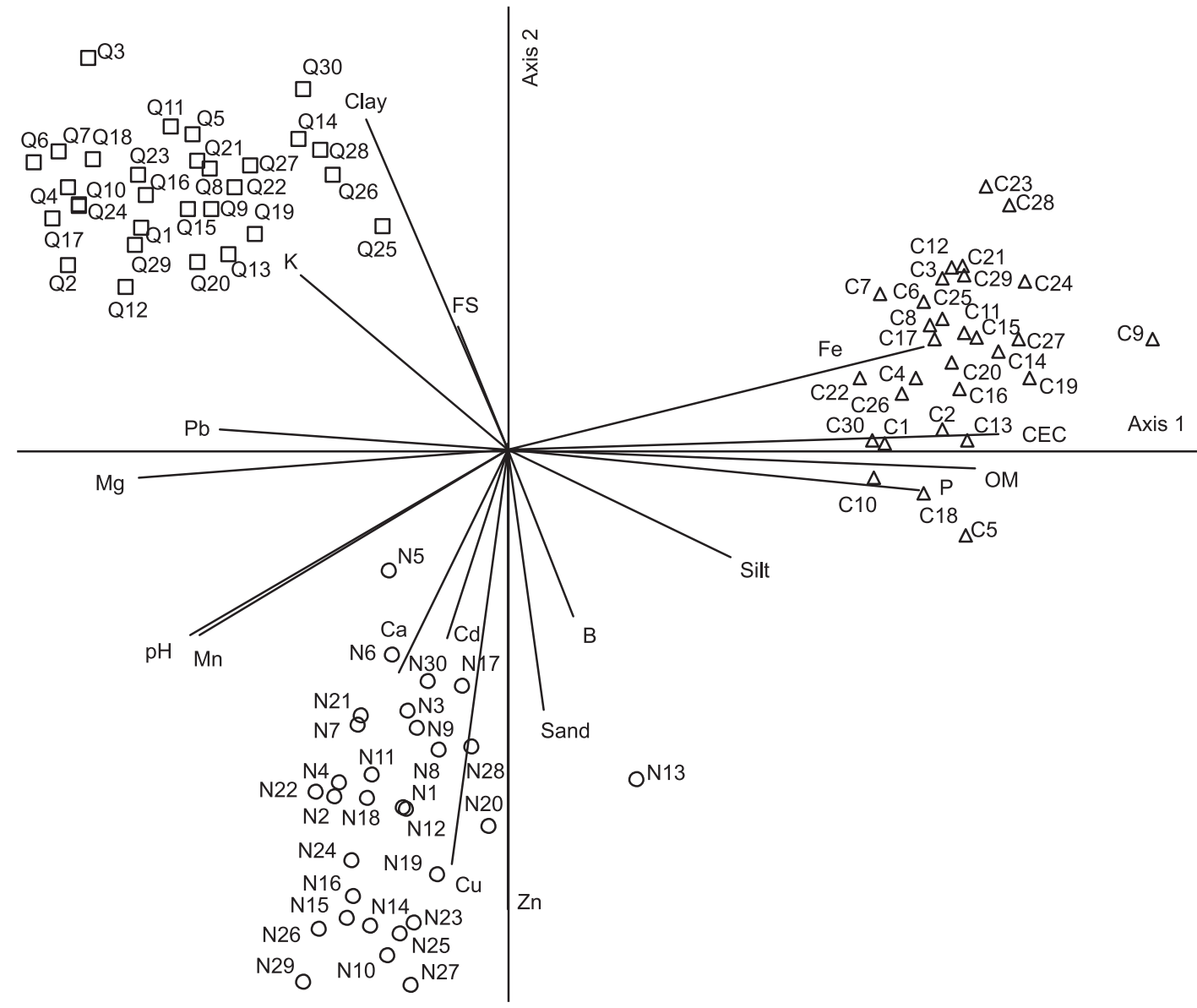

Figure 2. Diagram of ordination in the two first axes yielded by principal component analysis (PCA) of soil properties in the study areas: "canga couraçada" $(\mathrm{C})$, "canga nodular" $(\mathrm{N})$, and quartzitic "campo rupestre" $(\mathrm{Q})$. Soil properties are given as vectors. 
nodular soils. The second axis was negatively correlated mainly to $\mathrm{Zn}, \mathrm{Cu}$ and sand proportion, and positively correlated to clay proportion; this axis separates CNo from QCR. The "canga couraçada" quadrats have presented the highest levels of $\mathrm{Fe}, \mathrm{OM}$, and $\mathrm{P}$ and the highest values of $\mathrm{H}+\mathrm{Al}$ and $\mathrm{CEC}$; the "canga nodular" quadrats showed the highest levels of $\mathrm{Zn}, \mathrm{Cu}, \mathrm{Ca}$, and $\mathrm{Cd}$, while the quartzitic "campo rupestre" quadrats showed the highest levels of $\mathrm{K}, \mathrm{Pb}$, clay and fine soil proportion (FS) (table 1). Except for $\mathrm{Fe}$ and $\mathrm{Pb}, \mathrm{CNo}$ showed the highest levels of available metallic cations in soil.
Soil-plant species relationships - We found 40 species in "canga couraçada" (CCo), 131 in "canga nodular" (CNo), and 104 in quartzitic "campo rupestre" (QCR), totalling 228 species and 49 families. (R.C. Vincent et al., unpublished data).

116 species were selected for canonical correspondence analysis, belonging mainly to Asteraceae (24.1\%), Poaceae $(17.2 \%$ ) and Cyperaceae $(9.5 \%)$ (table 2). The sites were clearly segregated in CCA (figure 3 ) as also observed in PCA (figure 2). The eigenvalues of two first axis of CCA diagram were high (Axis $1=0.801$;

Table 2. Species selected for canonical correspondence analysis (CCA). Abbrev. = abbreviations used on figure 3 to species names. $\left(\mathrm{AD}=\right.$ absolute density $\left(\right.$ ind $\left.\mathrm{m}^{-2}\right) ; \mathrm{CCo}=$ "canga couraçada"; $\mathrm{CNo}=$ "canga nodular"; $\mathrm{QCR}=$ "campo rupestre"; $\mathrm{UN}=$ undetermined species). Collector's number of R. C. Vincent; voucher material deposited in BHCB* and ESA herbaria.

\begin{tabular}{|c|c|c|c|c|c|c|}
\hline \multirow{2}{*}{ Abbrev. } & \multirow{2}{*}{ Species } & \multirow{2}{*}{ Family } & \multirow{2}{*}{ Voucher } & \multicolumn{3}{|c|}{$\mathrm{AD}$} \\
\hline & & & & $\mathrm{CCo}$ & CNo & QCR \\
\hline Aam & Ayapana amygdalina (Lam.) R. M. King \& H. Rob. & Asteraceae & $581 *$ & - & - & 1.80 \\
\hline Ac1 & Acanthaceae 1 & Acanthaceae & 666 & - & - & 1.00 \\
\hline Ach & Axonopus chrysoblepharis (Lag.) Chase & Poaceae & 363 & - & - & 31.53 \\
\hline Afa & Ageratum fastigiatum (Gardner) R. M. King \& H. Rob. & Asteraceae & $465^{*}$ & - & 1.32 & 0.07 \\
\hline Agl & $\begin{array}{l}\text { Arthrocereus glaziovii (K. Schum.) N. P. Taylor } \\
\text { \& Zappi }\end{array}$ & Cactaceae & $21^{*}$ & 0.23 & - & - \\
\hline Ain & Andropogon ingratus Hack. & Poaceae & $729 *$ & 1.77 & 11.07 & 32.53 \\
\hline Amy & $\begin{array}{l}\text { Ageratum myriadenium (Sch. Bip. ex Baker) R. M. } \\
\text { King \& H. Rob. }\end{array}$ & Asteraceae & $8^{*}$ & 0.53 & - & - \\
\hline Apl & Alstroemeria plantaginea Mart. & Alstroemeriaceae & 329 & 0.17 & - & - \\
\hline Apr & Axonopus cf. pressus (Nees ex Steud.) Parodi & Poaceae & $131^{*}$ & - & 13.70 & - \\
\hline Are & Aristida recurvata Kunth & Poaceae & $550^{*}$ & - & 0.48 & 2.73 \\
\hline As2 & Aspilia sp. & Asteraceae & 188 & - & 0.15 & 6.60 \\
\hline Asi & Axonopus siccus (Nees) Kuhlm. & Poaceae & $543^{*}$ & 0.42 & 18.08 & 126.40 \\
\hline Asp & Achyrocline sp. & Asteraceae & 162 & - & 0.37 & - \\
\hline Ate & Acianthera teres (Lindl.) Borba & Orchidaceae & 125 & 6.65 & - & - \\
\hline Bca & Borreria cf. capitata DC. & Rubiaceae & 392 & - & - & 5.13 \\
\hline Bju & Bulbostylis junciformis C. B. Clarke & Cyperaceae & 705 & - & 2.30 & 1.80 \\
\hline Bpa & Bulbostylis paradoxa Nees & Cyperaceae & 712 & 0.05 & 0.10 & 3.47 \\
\hline Bri & Baccharis riedelii Sch. Bip. ex Baker & Asteraceae & 627 & - & - & 13.00 \\
\hline Bs1 & Barbacenia sp. & Velloziaceae & 5 & 3.07 & - & - \\
\hline Bse & Baccharis serrulata Pers. & Asteraceae & 17 & 0.25 & - & - \\
\hline Bsp & Bulbostylis sp. & Cyperaceae & 552 & - & 0.20 & 16.40 \\
\hline Bsu & Baccharis subdentata DC. & Asteraceae & $166^{*}$ & 0.02 & 0.43 & 0.27 \\
\hline Bva & Byrsonima cf. variabilis A. Juss. & Malpighiaceae & 93 & - & 0.25 & - \\
\hline Cam & Calea cf. multiplinervia Less. & Asteraceae & 639 & - & 0.37 & 1.00 \\
\hline Can & Croton antisyphiliticus Mart. & Euphorbiaceae & 669 & - & - & 7.20 \\
\hline Cca & Croton campestris A. St.-Hil. & Euphorbiaceae & $230 *$ & - & 0.57 & - \\
\hline Cde & Chamaecrista desvauxii (Collad.) Killip & Fabaceae & $674 *$ & - & 0.17 & - \\
\hline Chc & Chromolaena campestris (DC.) R. M. King \& H. Rob. & Asteraceae & 641 & - & - & 8.53 \\
\hline Che & Chrysolaena herbacea (Vell.) H. Rob. & Asteraceae & 610 & - & 0.17 & - \\
\hline
\end{tabular}


continuation

\begin{tabular}{|c|c|c|c|c|c|c|}
\hline \multirow{2}{*}{ Abbrev. } & \multirow{2}{*}{ Species } & \multirow{2}{*}{ Family } & \multirow{2}{*}{ Voucher } & \multicolumn{3}{|c|}{$\mathrm{AD}$} \\
\hline & & & & $\mathrm{CCo}$ & CNo & QCR \\
\hline Chm & $\begin{array}{l}\text { Chamaecrista mucronata (Spreng.) H. S. Irwin \& } \\
\text { Barneby }\end{array}$ & Fabaceae & 78 & - & 0.90 & - \\
\hline Cin & Chaptalia integerrima (Vell.) Burkart & Asteraceae & 111 & - & 0.63 & 1.40 \\
\hline $\mathrm{Cmu}$ & $\begin{array}{l}\text { Chromolaena multiflosculosa (DC.) R. M. King \& } \\
\text { H. Rob. }\end{array}$ & Asteraceae & $609 *$ & 0.67 & 1.58 & - \\
\hline Cpi & Chaptalia piloselloides (Vahl) Baker & Asteraceae & 623 & - & 0.50 & 3.07 \\
\hline Cpo & Chamaesyce potentilloides (Boiss.) Croizat & Euphorbiaceae & 670 & - & 0.25 & 0.53 \\
\hline Cst & $\begin{array}{l}\text { Chromolaena cf. stachyophylla (Spreng.) R. M. King } \\
\text { \& H. Rob. }\end{array}$ & Asteraceae & $638^{*}$ & - & 0.58 & 0.07 \\
\hline Cth & Cuphea thymoides Cham. \& Schltdl. & Lythraceae & 74 & - & 2.58 & 4.60 \\
\hline Cy1 & Cyperaceae 1 & Cyperaceae & 107 & - & 9.30 & - \\
\hline Cy2 & Cyperaceae 2 & Cyperaceae & 724 & 0.13 & 0.62 & - \\
\hline Сy3 & Cyperaceae 3 & Cyperaceae & 725 & - & - & 1.67 \\
\hline Cy4 & Cyperaceae 4 & Cyperaceae & 726 & - & - & 8.13 \\
\hline Dco & Declieuxia cf. cordigera Mart. \& Zucc. & Rubiaceae & 690 & - & - & 4.47 \\
\hline Doe & Declieuxia oenanthioides Mart. \& Zucc. & Rubiaceae & $474 *$ & - & 0.40 & - \\
\hline Ds2 & Ditassa sp. 2 & Apocynaceae & 657 & - & - & 1.73 \\
\hline Ds3 & Ditassa sp. 3 & Apocynaceae & 658 & - & - & 1.73 \\
\hline Dsa & Dyckia cf. saxatilis $\mathrm{Mez}$ & Bromeliaceae & 20 & 0.05 & 0.40 & - \\
\hline Ein & Echinolaena inflexa (Poir.) Chase & Poaceae & $138^{*}$ & - & 7.57 & 39.07 \\
\hline Epo & Eragrostis polytricha Nees & Poaceae & 135 & - & 0.60 & - \\
\hline Ese & Epidendrum secundum Jacq. & Orchidaceae & $28 *$ & 0.20 & - & - \\
\hline Esp & Erythroxylum sp. & Erythroxylaceae & 769 & - & 0.52 & - \\
\hline $\mathrm{Fa} 1$ & Fabaceae 1 & Fabaceae & 770 & - & - & 0.93 \\
\hline Gan & Galianthe cf. angustifolia (Cham. \& Schltdl.) Cabral & Rubiaceae & 423 & 0.83 & - & - \\
\hline Gma & Galactia martii DC. & Fabaceae & $224 *$ & - & 4.17 & - \\
\hline Gre & Gaylussacia reticulata Mart. ex Meisn. & Ericaceae & 177 & - & 3.65 & - \\
\hline Gsp & Gomphrena sp. & Amaranthaceae & 662 & - & 0.23 & - \\
\hline Hli & Hyptis lippioides Pohl ex Benth. & Lamiaceae & 155 & - & 0.23 & 0.07 \\
\hline Hlu & Hyptis cf. lucida Pohl ex Benth. & Lamiaceae & 681 & - & 0.23 & - \\
\hline Hsp & Hippeastrum sp. & Amaryllidaceae & 254 & 0.33 & - & - \\
\hline Jca & Jacaranda caroba (Vell.) DC. & Bignoniaceae & 91 & - & 1.30 & 0.07 \\
\hline Kpu & Kielmeyera cf. pumila Pohl. & Clusiaceae & 471 & - & 0.20 & 0.07 \\
\hline Kva & Kielmeyera variabilis Mart. & Clusiaceae & 130 & - & 0.58 & - \\
\hline Lde & Lessingianthus desertorum (Mart. ex DC.) H. Rob. & Asteraceae & 223 & - & 0.20 & - \\
\hline Ler & Lychnophora ericoides Mart. & Asteraceae & $11^{*}$ & 0.93 & - & - \\
\hline Lgr & Lippia gracilis Phil. & Verbenaceae & 16 & 0.20 & - & - \\
\hline Lla & Leptocoryphium lanatum (Kunth.) Nees & Poaceae & 745 & - & - & 28.27 \\
\hline Lly & Lucilia lycopodioides (Less.) S. E. Freire & Asteraceae & $447 *$ & - & 0.67 & - \\
\hline Lps & Lessingianthus psilophyllus (DC.) H. Rob. & Asteraceae & $612 *$ & - & 0.50 & 1.53 \\
\hline Ls1 & Lessingianthus sp. 1 & Asteraceae & 89 & - & 1.55 & 0.53 \\
\hline Ls2 & Lessingianthus sp. 2 & Asteraceae & 633 & - & - & 15.60 \\
\hline Lsi & Lessingianthus simplex (Less.) H. Rob. & Asteraceae & $615^{*}$ & - & 6.25 & - \\
\hline Lsp & Lagenocarpus sp. & Cyperaceae & 714 & - & - & 5.67 \\
\hline Lxa & Leandra cf. xanthostachys Cogn. & Melastomataceae & $247 *$ & - & 0.17 & - \\
\hline Mas & Mandevilla sp. & Apocynaceae & 655 & - & - & 5.20 \\
\hline Mca & Mimosa calodendron Mart. ex Benth. & Fabaceae & $325^{*}$ & 0.30 & - & - \\
\hline
\end{tabular}




\begin{tabular}{|c|c|c|c|c|c|c|}
\hline \multirow{2}{*}{ Abbrev. } & \multirow{2}{*}{ Species } & \multirow{2}{*}{ Family } & \multirow{2}{*}{ Voucher } & \multicolumn{3}{|c|}{$\mathrm{AD}$} \\
\hline & & & & CCo & CNo & QCR \\
\hline Mis & Mikania sp. & Asteraceae & 771 & - & 0.18 & - \\
\hline Mmi & Melinis minutiflora P. Beauv. & Poaceae & 102 & 0.45 & 10.27 & - \\
\hline Mne & Mimosa cf. neuroloma Benth. & Fabaceae & $221 *$ & - & 0.22 & - \\
\hline Nru & Neomarica rupestris (Ravenna) Chukr & Iridaceae & 79 & - & 0.18 & - \\
\hline Pap & Paspalum polyphyllum Nees ex Trin. & Poaceae & $752 *$ & - & 1.90 & 2.40 \\
\hline Pch & Phyllanthus choretroides Müll. Arg. & Euphorbiaceae & 503 & - & - & 2.80 \\
\hline Pde & Peperomia decora Dahlst. & Piperaceae & 9 & 2.05 & - & - \\
\hline \multirow[t]{2}{*}{ Pep } & Peltaea cf. polymorpha (A. St.-Hil.) Krapov. \& & & & & & \\
\hline & Cristóbal & Malvaceae & 357 & - & 0.15 & 1.47 \\
\hline Pfs & Pfaffia sp. & Amaranthaceae & 664 & - & 0.40 & - \\
\hline $\operatorname{Pgn}$ & Pfaffia gnaphaloides (L. f.) Mart. & Amaranthaceae & 123 & - & 2.88 & - \\
\hline Pme & Periandra mediterranea (Vell.) Taub. & Fabaceae & 68 & - & 0.25 & - \\
\hline Pni & Phyllanthus niruri L. & Euphorbiaceae & $668 *$ & - & 3.53 & - \\
\hline Po1 & Poaceae 1 & Poaceae & 766 & - & - & 30.93 \\
\hline $\mathrm{Po} 2$ & Poaceae 2 & Poaceae & 765 & - & - & 1.73 \\
\hline Po3 & Poaceae 3 & Poaceae & 767 & - & - & 2.53 \\
\hline Po4 & Poaceae 4 & Poaceae & 768 & - & - & 4.80 \\
\hline Po5 & Poaceae 5 & Poaceae & 772 & - & 0.32 & - \\
\hline \multirow[t]{2}{*}{ Ppo } & Pseudogynoxys pohlii (Sch. Bip. ex Baker) & & & & & \\
\hline & Leitão Filho & Asteraceae & 168 & - & 2.18 & - \\
\hline Psc & Paspalum scalare Trin. & Poaceae & 753 & 1.97 & 2.48 & - \\
\hline Psp & Paspalum sp. & Poaceae & 746 & - & 0.32 & 1.00 \\
\hline Pto & Peixotoa tomentosa A. Juss. & Malpighiaceae & 66 & - & 0.32 & 0.13 \\
\hline Pur & Polygala cf. urbanii Chodat & Polygalaceae & 683 & - & 0.50 & 0.07 \\
\hline Ran & Rhynchospora andina Kük. & Cyperaceae & 717 & - & - & 33.27 \\
\hline Rco & Rhynchospora consanguinea (Kunth.) Boeck. & Cyperaceae & $229 *$ & - & 0.38 & 0.60 \\
\hline Rs1 & Rhynchospora sp. & Cyperaceae & $553 *$ & - & 0.17 & - \\
\hline Rsp & Ruellia sp. & Acanthaceae & 121 & - & 0.38 & - \\
\hline Sbr & $\begin{array}{l}\text { Symphyopappus brasiliensis (Gardner) R. M. King } \\
\text { \& H. Rob. }\end{array}$ & Asteraceae & 18 & 0.43 & - & - \\
\hline Sca & $\begin{array}{l}\text { Sophronitis caulescens (Lindl.) Van den Berg \& } \\
\text { M. W. Chase }\end{array}$ & Orchidaceae & 1 & 9.42 & - & - \\
\hline $\mathrm{Sgl}$ & Stachytarpheta glabra Cham. & Verbenaceae & 12 & 0.52 & - & - \\
\hline Sma & Sisyrinchium marchio Steud. & Iridaceae & 699 & - & - & 2.07 \\
\hline Ssa & Schizachyrium sanguineum (Retz.) Alston & Poaceae & $758 *$ & - & 0.32 & 40.47 \\
\hline Ssp & Symphyopappus sp. & Asteraceae & 640 & - & - & 4.93 \\
\hline Sur & Stevia urticifolia Thunb. & Asteraceae & $475^{*}$ & - & 0.25 & 0.20 \\
\hline Tmu & Tibouchina multiflora Cogn. & Melastomataceae & $19 *$ & 0.20 & - & - \\
\hline Tob & Turnera oblongifolia Cambess. & Turneraceae & 434 & - & 0.63 & 2.40 \\
\hline Tre & Thrasyopsis repanda (Nees) Parodi & Poaceae & 764 & - & - & 14.20 \\
\hline Tsp & Trachypogon spicatus Kuntze & Poaceae & 105 & - & 57.33 & 0.07 \\
\hline Un2 & UN sp. 2 & UN & 773 & - & 0.22 & - \\
\hline Un4 & UN sp. 4 & $\mathrm{UN}$ & 774 & - & - & 3.73 \\
\hline Un7 & UN sp. 7 & UN & 704 & - & - & 2.13 \\
\hline $\mathrm{Vku}$ & Viguiera kunthiana Gardner & Asteraceae & 622 & - & - & 3.00 \\
\hline Vre & Vellozia cf. resinosa Mart. ex Schult. f. & Velloziaceae & $4 *$ & 3.38 & - & - \\
\hline Zdi & Zornia cf. diphylla (L.) Pers. & Fabaceae & 676 & - & 0.02 & 2.33 \\
\hline
\end{tabular}



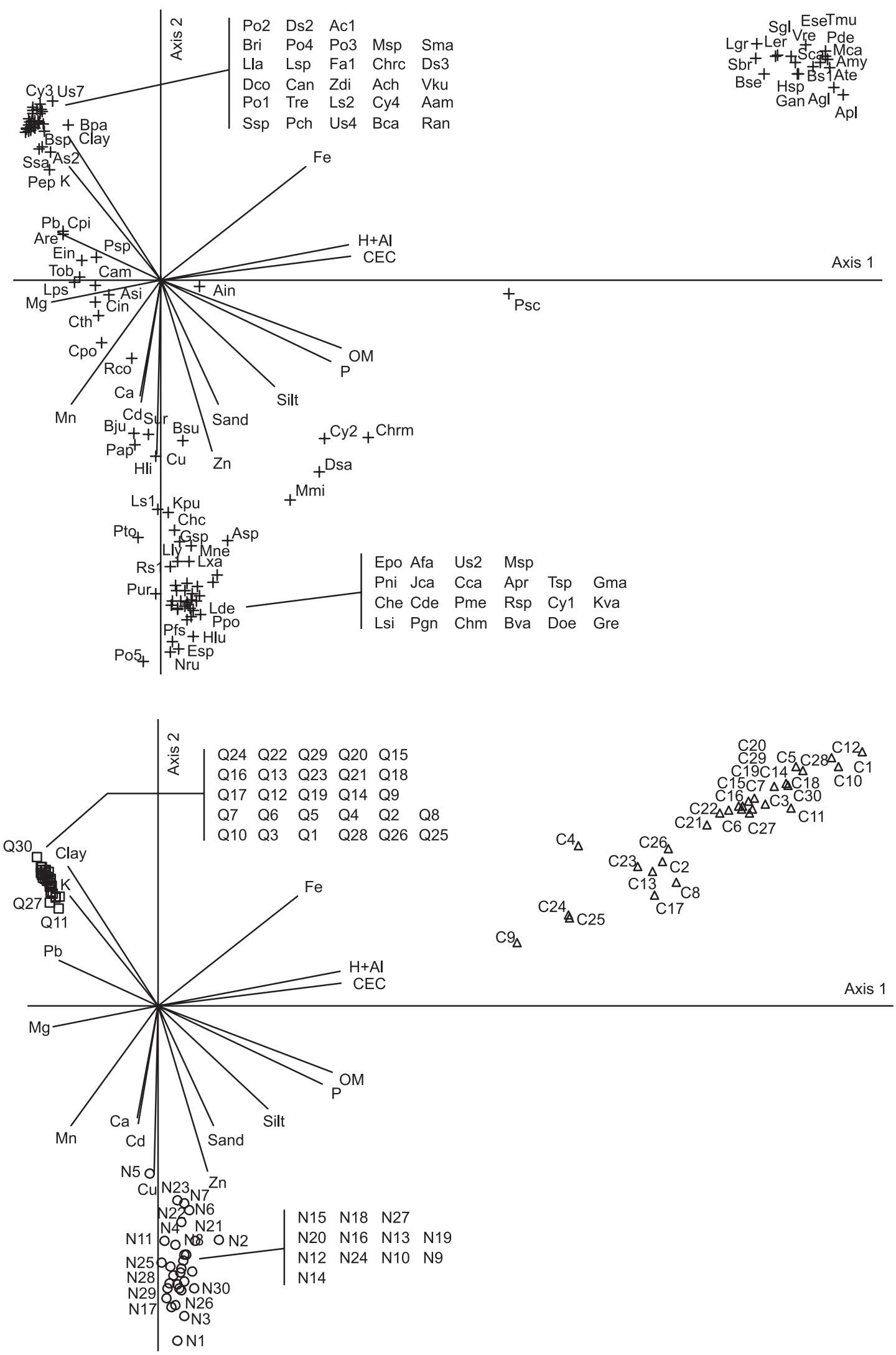

Figure 3. Diagram of ordination in the two first axes yielded by canonical correspondence analysis (CCA) of soil properties and plant species densities in "canga couraçada" (C), "canga nodular" (N) and quartzitic "campo rupestre" (Q). A. Species; B. Quadrats. Soil properties are given as vectors. Full names of species are given in the table 2. 
Axis $2=0.628$ ), the first one explaining $18.8 \%$ of total variance and the second $14.7 \%$ (table 3 ). The Pearson's correlation index obtained from Monte Carlo permutation test showed that plant abundance was significantly correlated with soil properties $(r=0.983, P<0.01$ in the first axis; $r=0.970, P<0.01$ in the second one). The first axis was positively correlated to $\mathrm{CEC}, \mathrm{H}+\mathrm{Al}, \mathrm{OM}, \mathrm{P}$ and $\mathrm{Fe}$, and negatively correlated with $\mathrm{Mg}$ and $\mathrm{Pb}$ levels; this axis separates CCo from QCR and CNo. The second axis showed strong negative correlation with $\mathrm{Cu}, \mathrm{Zn}$, besides $\mathrm{Mn}, \mathrm{Cd}, \mathrm{Ca}$ and sand, and positive correlation with clay and $\mathrm{K}$, and has separated $\mathrm{CNo}$ from $\mathrm{CCo}$ and QCR (figure 3b).

The dispersion of quadrats in CCA diagram reveals a gradient in $\mathrm{CCo}$ (figure $3 \mathrm{~b}$ ). A group of quadrats showed high abundance of individuals of species found only in this area (upper right of the figure 3a), mainly Barbacenia sp.1 and Vellozia cf. resinosa (Velloziaceae), and Acianthera teres and Sophronitis caulescens (Orchidaceae), while the remnant quadrats showed lower densities of the exclusive species and more individuals of species common to the "canga nodular", such as Paspalum scalare and Melinis minutiflora (Poaceae), Chromolaena multiflosculosa (Asteraceae), Cyperaceae 2, and Dyckia cf. saxatilis (Bromeliaceae) (figure 3a). In CNo, we also observed such a gradient but not so intense, while the QCR quadrats were strongly aggregated in the CCA diagram (figure 3b). A gradient between QCR and $\mathrm{CNo}$ was observed in figure $3 \mathrm{a}$, where exclusive species have occupied the ends of gradient and the common ones have occupied intermediary positions in the diagram according to their abundances, mainly Chaptalia piloselloides, C. integerrima and Lessingianthus

Table 3. Summary of canonical correspondence analysis (CCA) and Monte Carlo permutation test of plant species densities and soil properties for "canga couraçada", "canga nodular" and quartzitic "campo rupestre".

\begin{tabular}{lccc}
\hline & Axis 1 & Axis 2 & Axis 3 \\
\hline $\begin{array}{l}\text { Eigenvalue } \\
\begin{array}{l}\text { Percentage of variance } \\
\text { explained }\end{array}\end{array}$ & 0.801 & 0.628 & 0.086 \\
$\begin{array}{l}\text { Cumulative percentage of } \\
\text { variance }\end{array}$ & 18.8 & 14.7 & 2.0 \\
$\begin{array}{l}\text { Pearson's correlation for } \\
\text { species-soil }\end{array}$ & 0.983 & 0.970 & 0.896 \\
$\begin{array}{l}\text { Significance of correlation } \\
\text { (Monte Carlo test) }\end{array}$ & 0.01 & 0.01 & 0.01 \\
\hline
\end{tabular}

psilophyllus (Asteraceae), Aristida recurvata and Echinolaena inflexa (Poaceae), Turnera oblongifolia (Turneraceae), and Cuphea thymoides (Lythraceae). Among the species common to the three sites, Andropogon ingratus and Axonopus siccus (Poaceae) have showed higher densities in CNo and QCR (table 2) occupying the centre of CCA diagram, while Baccharis subdentata (Asteraceae) and Bulbostylis paradoxa have showed higher affinity to CNo and QCR soils respectively (figure 3a, table 2).

\section{Discussion}

Soils from the ferruginous "campos rupestres" and the quartzitic "campo rupestre" studied here are similar to those of other quartzitic "campos rupestres" concerning to low pH, P levels and base saturation (Vitta 1995, Conceição \& Giulietti 2002, Teixeira \& Lemos-Filho 2002). The highest levels of most of metallic cations in CNo was expected since intemperization releases elements from rock. The high acidity of soil may enhance availability of metal cations toxic to plants, except for copper, because $\mathrm{H}^{+}$and $\mathrm{Cu}$ antagonism may reduce copper toxicity (Malavolta et al. 1967, Levitt 1980, Bornemisza 1982). Thus, except for this cation, the high concentration of heavy metals in the acidic soils of the study sites probably acts as an additional stress factor to their plants. The quartzitic fields where QCR is situated are embedded among ferruginous formations, probably explaining the high $\mathrm{Pb}, \mathrm{Mn}, \mathrm{Cu}$, and $\mathrm{Fe}$ concentrations found, as recorded by Teixeira \& Lemos-Filho (1998) in soils of a quartzitic rocky outcrop in Serra de Itabirito, a range also situated in "Quadrilátero Ferrífero".

The high OM content found in CCo soil (table 1), although partially explained by the high proportion of not decomposed plant debris, may reduce both the availability and the toxicity of $\mathrm{Zn}$ to plants (Malavolta et al. 1967, Levitt 1980, Bornemisza 1982).

The Fe content found in CCo is similar to that found at "Serra dos Carajás" (northern Brazil), where the values are 435.3 and $589.6 \mathrm{mg} \mathrm{dm}^{-3}$ (Silva 1992). At "Serra do Itabirito", in "Quadrilátero Ferrífero", Teixeira \& LemosFilho (2002) found $\mathrm{Fe}$ amounts similar to CNo values, both in ferruginous and quartzitic outcrops, respectively 151.5 and $171.0 \mathrm{mg} \mathrm{dm}^{-3}$, while the value found in southeastern "Quadrilátero Ferrífero" (Gonçalves-Alvim $\&$ Fernandes 2001) was lower than any other ferruginous outcrop (74.0 $\mathrm{mg} \mathrm{dm}^{-3}$ ). The comparison of other metal levels among ferruginous outcrops from "Cadeia do Espinhaço" and "Serra dos Carajás" reveals that CCo have the highest amounts of $\mathrm{Cu}$ and $\mathrm{Zn}$, but less $\mathrm{Mn}, \mathrm{Cr}$ 
and Cd (Silva 1992, Gonçalves-Alvim \& Fernandes 2001, Teixeira \& Lemos-Filho 2002). In ultramafic soils at Niquelândia (central Brazil) the total amounts of $\mathrm{Cr}, \mathrm{Cu}$, $\mathrm{Mn}$ and Ni were very high (Reeves et al. 2007), but it is not possible to compare them to those found here due to the employed method, since they determined total amounts, while the present study deals only with available content.

The study sites were different edaphically, floristically and phytosociologically, as evidenced by ordination analyses (figures 2 and 3). The main edaphic variables influencing site segregation are related to soil fertility, such as $\mathrm{CEC}, \mathrm{P}$ and $\mathrm{Mg}$ levels, $\mathrm{H}+\mathrm{Al}, \mathrm{OM}$ content, clay, and to the amounts of metallic cations, mainly $\mathrm{Fe}, \mathrm{Pb}$, $\mathrm{Zn}$, and $\mathrm{Cu}$ (figure 2).

Some authors have related the physiognomical gradient of "cerrado" to soil fertility (Alvim \& Araújo 1952, Goodland 1979), while others did not find such relationship (Gibbs et al. 1983, Ruggiero et al. 2002). This absence of relationship, however, could be explained by the choice of the stratum analyzed; the superficial root systems of many "cerrado" herbs and subshrubs allow higher exploration of superficial soil horizon by these plants than by the woody component (Walker 1984). Thus, spatial edaphic and microtopographic variability of superficial soil produce more edaphic microsites for herbaceous and subshrub species than for woody species, which may respond to a combination of those microsites (Sagers \& Lyon 1997, Vieira 1997). Additionally, herbaceous and subshrub flora of "cerrado" have two or three times the number of species of the woody flora (Mantovani \& Martins 1993, Castro et al. 1999); a relation of 1.7:1 was observed in a phytosociological survey performed in both strata of an 1 ha area of "campo cerrado" in São Paulo State (Vincent et al. 1992). In same way "campos rupestres" present such spatial heterogeneity since there are areas with exposed rock (outcrops), stony or nodular soils or primary soils, besides edaphic and microtopographic variability characteristic of the superficial soil. Although "cerrado" soils are deeper than "campo rupestre" ones, their higher variability occurs in superficial soil, then it is reasonable to expect high correlation between soil properties and the lower layer of vegetation. The spatial edaphic heterogeneity together with the high species diversity of herbaceous-subshrub layer make this stratum more useful to verify factors affecting physiognomical gradient in "cerrado" or in "campos rupestres" as suggested by data obtained here (figure 3 ).

The CCo quadrat gradient observed in CCA diagram (figure 3) must not be attributed to the soil variables analyzed here, since this pattern was not observed in PCA diagram (figure 2). Probably, this is a result of a gradient of exposed rock proportion, since the quadrats with higher density of epilithic species clumped together. On the other hand, the gradient in CNo quadrats reflects the gradient of heavy metal levels, especially $\mathrm{Cu}, \mathrm{Zn}$, and $\mathrm{Cd}$ (figures 2, 3). High levels of $\mathrm{Cu}, \mathrm{Ni}$, and $\mathrm{Co}$ on metalliferous soils in Rhodesia were correlated with floristic composition and with the density of populations of Becium hombley and B. obovatum (Lamiaceae) (Howard-Williams 1970). The distribution of QCR quadrats and species reveals a correlation between the group of species exclusive to QCR and soil, while the species shared by QCR and CNo grow on soils with intermediate properties.

Although sharing a common geological origin, the "canga couraçada" and the "canga nodular" showed quite different soil properties probably due to the effect of rock degradation on release of mineral particles and chemical elements, besides the differences in exposed rock proportion. On the other hand, although CNo and QCR have nodular substrates the different geological origins play an important role in soil differences. The data presented here have showed the influence of some soil properties on the abundance and distribution of species within and among areas.

Acknowledgements - We thank Jaime Bertoluci for comments on the manuscript and for helping in the fieldwork, Andrea de Souza and Jane M.Q. Alves for helping in the fieldwork, João Renato Stehmann, Marcos Sobral, Jimi Nakagima, Pedro L. Viana, Rubens Mota and Lindolpho Capellari Junior for species identification, and Instituto Estadual de Florestas (IEF/MG) and Copasa for permit access to the areas. We also thank the anonymous referees for suggestions to manuscript. R.C. Vincent have received a grant from Capes.

\section{References}

ALVIM, P.T. \& ARAÚJO, W.A. 1952. El suelo como factor ecológico en el desarrollo de la vegetación en el centrooeste del Brasil. Turrialba 2:153-160.

ANTONOVICS, J., BRADSHAW, A.D. \& TURNER, R.G. 1971. Heavy metal tolerance in plants. Advances in Ecological Research 7:1-85.

BORNEMISZA, E. 1982. Introducción a la química de suelos. (Monografias Científicas, Série de Química, 25). OEA, Washington, DC.

CASTRO, A.A.J.F., MARTINS, F.R., TAMASHIRO, J.Y. \& SHEPHERD, G.J. 1999. How rich is the flora of Brazilian cerrados? Annals of the Missouri Botanical Garden 86: 192-224.

CONCEIÇÃO, A.A. \& GIULIETTI, A.M. 2002. Composição florística e aspectos estruturais de campo rupestre em dois platôs no Morro do Pai Inácio, Chapada Diamantina, Bahia, Brasil. Hoehnea 29:37-48. 
COUTINHO, L.M. 1978. O conceito de cerrado. Revista Brasileira de Botânica 1:17-23.

DRUMMOND, G.M., MARTINS, C.S., MACHADO, A.B.M., SEBAIO, F.A. \& ANTONINI, Y. (orgs.) 2005. Biodiversidade em Minas Gerais - Um atlas para sua conservação. $2^{\text {nd }}$ ed. Fundação Biodiversitas, Belo Horizonte.

DUVIGNEAUD, P. \& DENAEYER-DE SMET, S. 1960. Action de certains métaux lourds du sol (cuivre, cobalt, manganèse, uranium) sur la végétation du Haut-Katanga. In Rapports du sol et de la végétation (G. ViennotBourgin, ed.). Masson et Cie., Paris, p.121-139.

GIBBS, P., LEITÃO-FILHO, H.F. \& SHEPHERD, G.J. 1983. Floristic composition and community structure in an area of cerrado in SE Brazil. Flora 173:433-449.

GIUlieTti, A.M., MENEZES, N.L., PIRANI, J.R., MEGURO, M. \& WANDERLEY, M.G.L. 1987. Flora da Serra do Cipó, Minas Gerais: caracterização e lista das espécies. Boletim de Botânica da Universidade de São Paulo 9:1-151.

GIULIETTI, A.M., PIRANI, J.R. \& HARLEY, R.M. 1997. Espinhaço range region - eastern Brazil. In Centres of plant diversity - a guide and strategy for their conservation, The Americas (S.D. Davis, V.H. Heywood, O. Herrera-MacBryde, J. Villa-Lobos \& A.C. Hamilton, eds.). WWF/IUCN. IUCN Publications Unit, Cambridge, v.3, p.397-404.

GOODLAND, R. 1979. Análise ecológica da vegetação do cerrado. In Ecologia do cerrado (R. Goodland \& M.G. Ferri, eds.). Itatiaia/EDUSP, Belo Horizonte/São Paulo, p.61-186.

GONÇALVES-ALVIM, S.J. \& FERNANDES, G.W. 2001. Biodiversity of galling insects: historical, community and habitat effects in four neotropical savannas. Biodiversity and Conservation 10:79-98.

HERZ, N. 1978. Metamorphic rocks of the Quadrilátero Ferrífero, Minas Gerais, Brazil. Geological Survey Professional Paper 641:1-81.

HOWARD-WILLIAMS, C. 1970. The ecology of Becium hombley in Central Africa with special reference to metalliferous soils. Journal of Ecology 58:745-763.

JACOBI, C.M., CARMO, F.F., VINCENT, R.C. \& STEHMANN, J.R. 2007. Plant communities on ironstone outcrops: a diverse and endangered Brazilian ecosystem. Biodiversity and Conservation 16:2185-2200.

KÖHL, K.I. 1997. Do Armeria maritima (Mill.) Willd. ecotypes from metalliferous soils and non-metalliferous soils differ in growth response under Zn stress? A comparison by a new artificial soil method. Journal of Experimental Botany 48:1959-1967.

LEVITT, J. 1980. Responses of plants to environmental stresses. v.II. Water, radiation, salt, and other stresses. Academic Press, New York.

MAGALHÃES, G.M. 1966. Sobre os cerrados de Minas Gerais. Anais da Academia Brasileira de Ciências 38:59-70.
MALAVOLTA, E., HAAG, H.P., MELLO, F.A.F. \& BRASILSOBRINHO, M.O.C. 1967. Nutrição mineral de algumas culturas tropicais. Pioneira/EDUSP, São Paulo.

MANTOVANI, W. \& MARTINS, F.R. 1993. Florística do cerrado na Reserva Biológica de Moji Guaçu, SP. Acta Botanica Brasilica 7:33-60.

McCUNE, B. \& MEFFORD, M.J. 1999. Multivariate analysis of ecological data. Version 4.14. (PCOrd). MjM Software, Oregon.

MORELLATO, P.C. \& ROSA, N.A. 1991. Caracterização de alguns tipos de vegetação na região amazônica, Serra dos Carajás, Pará, Brasil. Revista Brasileira de Botânica 14:1-14.

MUELLER-DOMBOIS, D. \& ELLENBERG, H. 1974. Aims and methods of vegetation ecology. Willey and Sons, New York.

OLIVEIRA-FILHO, A.T., CURI, N., VILELA, E.A. \& CARVALHO, D.A. 2001. Variation in tree community composition and structure with changes in soil properties within a fragment of semideciduous forest in South-Eastern Brazil. Edinburgh Journal of Botany 58:139-158.

POMERENE, J.B. 1964. Geology and ore deposits of the Belo Horizonte, Ibirité, and Macacos quadrangles, Minas Gerais, Brazil. Geological Survey Professional Paper 341:1-84.

PORTO, M.L. 1989. Tolerância ao cobre em ecótipos de Schinus lentiscifolius (Anacardiaceae) de áreas de mineração no Rio Grande do Sul, Brasil. Acta Botanica Brasilica 3:23-31.

PORTO, M.L. \& SILVA, M.F.F. 1989. Tipos de vegetação metalófila em áreas da Serra de Carajás e de Minas Gerais. Acta Botanica Brasilica 3:13-21.

PRODEMGE, Minas Gerais. 1996. Atlas de zoneamento agroclimático do estado de Minas Gerais. In www.geominas.mg.gov.br (acessed 2005 February 20).

REEVES, R.D., BAKER, A.J.M., BECQUER, T., ECHEVARRIA, G. \& MIRANDA, Z.J.G. 2007. The flora and biogeochemistry of the ultramafic soils of Goiás state, Brazil. Plant Soil 293:107-119.

RIZZINI, C.T. 1997. Tratado de fitogeografia do Brasil. Âmbito Cultural, Rio de Janeiro.

RUGGIERO, P.G.C., BATALHA, M.A., PIVELLO, V.R. \& MEIRELLES, S.T. 2002. Soil-vegetation relationships in cerrado (Brazilian savanna) and semideciduous forest, southeastern Brazil. Plant Ecology 160:1-16.

SAGERS, C.L. \& LYON, J. 1997. Gradient analysis in a riparian landscape: contrasts among forest layers. Forest Ecology and Management 96:13-26.

SECCO, R.S. \& MESQUITA, A.I. 1983. Notas sobre a vegetação de canga da Serra Norte - I. Boletim do Museu Paraense Emílio Goeldi, nova série Botânica 59:1-13.

SIEGEL, S. 1956. Nonparametric statistics for the behavioral sciences. McGraw-Hill, New York. 
SILVA, M.F.F. 1991. Análise florística da vegetação que cresce sobre canga hematítica em Carajás - Pará (Brasil). Boletim do Museu Paraense Emílio Goeldi, série Botânica 7:79-105.

SILVA, M.F.F. 1992. Distribuição de metais pesados na vegetação metalófila de Carajás. Acta Botanica Brasilica 6:107-22.

SILVA, M.F.F. \& ROSA, N.A. 1990. Estudos botânicos na área do Projeto Ferro Carajás - Serra Norte. I. Aspectos fito-ecológicos dos campos rupestres. In Anais do XXXV Congresso Nacional de Botânica (1984). SBB, Manaus, p.367-379.

SILVA, M.F.F., SECCO, R.S. \& LOBO, M.G.A. 1996. Aspectos ecológicos da vegetação rupestre da Serra dos Carajás, estado do Pará, Brasil. Acta Amazonica 26:17-44.

SIMMONS, G.C. 1960. Origin of certain cangas of the "Quadrilátero Ferrífero" of Minas Gerais, Brazil. Boletim da Sociedade Brasileira de Geologia 9:37-59.

SIMMONS, G.C. 1968. Geology and iron deposits of the Western Serra do Curral, Minas Gerais, Brazil. Geological Survey Professional Paper 341:1-57.

STATSOFT, Inc. 2001. Statistica (Data analysis software system), version 6.

TEIXEIRA, W.A. \& LEMOS-FILHO, J.P. 1998. Metais pesados em folhas de espécies lenhosas colonizadoras de uma área de mineração de ferro em Itabirito, Minas Gerais. Revista Árvore 22:381-388.

TEIXEIRA, W.A. \& LEMOS-FILHO, J.P. 2002. Fatores edáficos e a colonização de espécies lenhosas em uma cava de mineração de ferro em Itabirito, Minas Gerais. Revista Árvore 26:25-33.
TER BRAAK, C.J.F. 1995. Ordination. In Data analysis in community and landscape ecology (R.H.G. Jongman, C.J.F. ter Braak \& O.F.R. Van Tongeren, eds.). Cambridge Univ. Press, Cambridge, p.91-173.

TER BRAAK, C.F.J. \& LOOMAN, C.W.N. 1995. Regression. In Data analysis in community and landscape ecology (R.H.G. Jongman, C.J.F. ter Braak \& O.F.R. Van Tongeren, eds.). Cambridge Univ. Press, Cambridge. p.29-77.

VIANA, P.L. \& LOMBARDI, J.A. 2007. Florística e caracterização dos campos rupestres sobre canga na Serra da Calçada, Minas Gerais, Brasil. Rodriguésia 58:159-177.

VIEIRA, S.R. 1997. Variabilidade espacial de argila, silte e atributos químicos em uma parcela experimental de um latossolo roxo em Campinas (SP). Bragantia 56:181-190.

VINCENT, R.C., JACOBI, C.M. \& ANTONINI, Y. 2002. Diversidade na adversidade. Ciência Hoje 31:64-67.

VINCENT, R.C., MIYAZAKI, S.L., GOMES, E.P.C. \& MANTOVANI, W. 1992. Estrutura e composição florística do cerrado de Emas, Pirassununga, SP. In Anais do $8^{\circ}$ Congresso da SBSP. SBSP, Campinas, p.139-151.

VITTA, F.A. 1995. Composição florística e ecologia de comunidades campestres na Serra do Cipó, Minas Gerais. Dissertação de Mestrado, Universidade de São Paulo, São Paulo.

WALKER, B.H. 1984. Structure and function of savannas: an overview. In Ecology and management of the world's savannas (J.C. Tohill \& J.J. Mott, eds.). The Australian Academy of Science, Canberra, p.83-91.

ZAR, J.H. 1996. Biostatistical analysis. Prentice Hall, New Jersey. 